\title{
Results of a Si/CdTe Compton Telescope
}

\author{
Kousuke Oonuki $^{a, b}$, Takaaki Tanaka $^{a, b}$, Shin Watanabe ${ }^{a}$, Shin'ichiro Takeda ${ }^{a, b}$, \\ Kazuhiro Nakazawa $^{a}$, Takefumi Mitani ${ }^{a, b}$, Tadayuki Takahashi ${ }^{a, b}$, Hiroyasu Tajima $^{c}$, \\ Yasushi Fukazawa ${ }^{d}$, Masaharu Nomachi ${ }^{e}$ \\ ${ }^{a}$ Institute of Space and Astronautical Science (ISAS/JAXA), Sagamihara, Kanagawa \\ 229-8510, Japan \\ ${ }^{b}$ Department of Physics, University of Tokyo, Bunkyo, Tokyo 113-0033, Japan \\ ${ }^{c}$ Stanford Linear Accelerator Center, Stanford, CA 94309-4349 \\ ${ }^{d}$ Department of Physics, Hiroshima University, Higashi-Hiroshima, 739-8526, Japan \\ eDepartment of Physics, Osaka University, Toyonaka, Osaka, 560-0043, Japan
}

\begin{abstract}
We have been developing a semiconductor Compton telescope to explore the universe in the energy band from several tens of $\mathrm{keV}$ to a few $\mathrm{MeV}$. We use a Si strip and CdTe pixel detector for the Compton telescope to cover an energy range from $60 \mathrm{keV}$. For energies above several hundred keV, the higher efficiency of CdTe semiconductor in comparison with $\mathrm{Si}$ is expected to play an important role as an absorber and a scatterer. In order to demonstrate the spectral and imaging capability of a CdTe-based Compton Telescope, we have developed a Compton telescope consisting of a stack of CdTe pixel detectors as a small scale prototype. With this prototype, we succeeded in reconstructing images and spectra by solving the Compton equation from $122 \mathrm{keV}$ to $662 \mathrm{keV}$. The energy resolution (FWHM) of reconstructed spectra is $7.3 \mathrm{keV}$ at $511 \mathrm{keV}$ and $3.1 \mathrm{keV}$ at $122 \mathrm{keV}$, respectively. The angular resolution obtained at $511 \mathrm{keV}$ is measured to be $12.2^{\circ}$ (FWHM).
\end{abstract}

Keywords: gamma-ray, Compton telescope, semiconductor, $\mathrm{CdTe}, \mathrm{Si}$

\section{INTRODUCTION}

The hard X-ray and gamma-ray bands ranging from several tens of $\mathrm{keV}$ to a few $\mathrm{MeV}$ are important windows for exploring the energetic universe. It is in these regions that high energy phenomena such as nucleosynthesis and particle acceleration become dominant. However, observation sensitivity in this energy band has been still limited, due to high background, low detection efficiency, and difficulty of imaging by means of focusing technology. A semiconductor Compton telescope utilizing the good energy and position resolution of semiconductors is one of the most promising ways to realize a breakthrough in this energy band.

Based on our recent achievements for high resolution CdTe and Si imaging detectors, ${ }^{1-5}$ we have proposed a $\mathrm{Si} / \mathrm{CdTe}$ semiconductor Compton telescope as a next generation Compton telescope. ${ }^{6,7}$ The basic concept of the $\mathrm{Si} / \mathrm{CdTe}$ Compton telescope is to utilize $\mathrm{Si}$ as a scatterer and CdTe as an absorber. Si works as a good scatterer with energies higher than $60 \mathrm{keV}$ due to large Compton scattering efficiency, and CdTe has high photo absorption efficiency as an absorber due to the large atomic numbers of $\mathrm{Cd}(\mathrm{Z}=48)$ and $\mathrm{Te}(\mathrm{Z}=52)$. The $\mathrm{Si} / \mathrm{CdTe}$ Compton telescope surpasses scintillator-based Compton telescopes by its high energy and angular resolution. According to the measurements with our prototype which is composed of six layers of Double-sided Si strip detector (DSSD) with a thickness of $300 \mu \mathrm{m}$ and CdTe pixel detectors with a thickness of $500 \mu \mathrm{m}$, we have successfully achieved Compton reconstructed images of gamma-rays from $81 \mathrm{keV}$ to $662 \mathrm{keV}$, and an angular resolution of $3.9^{\circ}$ for $511 \mathrm{keV}$ gamma-rays. ${ }^{8-10}$

In order to achieve much higher efficiency for gamma-rays above a few hundred $\mathrm{keV}$, we need a CdTe detector as thick as several $\mathrm{mm}$. One idea to achieve such a thick device is a stacked CdTe detector. ${ }^{11-13}$ At energies above $300 \mathrm{keV}$, the stacked CdTe detector by itself works as a Compton telescope. To understand the behavior of

Further author information: (Send correspondence to K.O.)

K.O.: E-mail: oonuki@astro.isas.jaxa.jp, Telephone: 81427598510

Contributed to SPIE Conference on Optics and Photonics 2005, 07/31-8/4/2005, San Diego, CA

Work supported in part by Department of Energy contract DE-AC02-76SF00515 

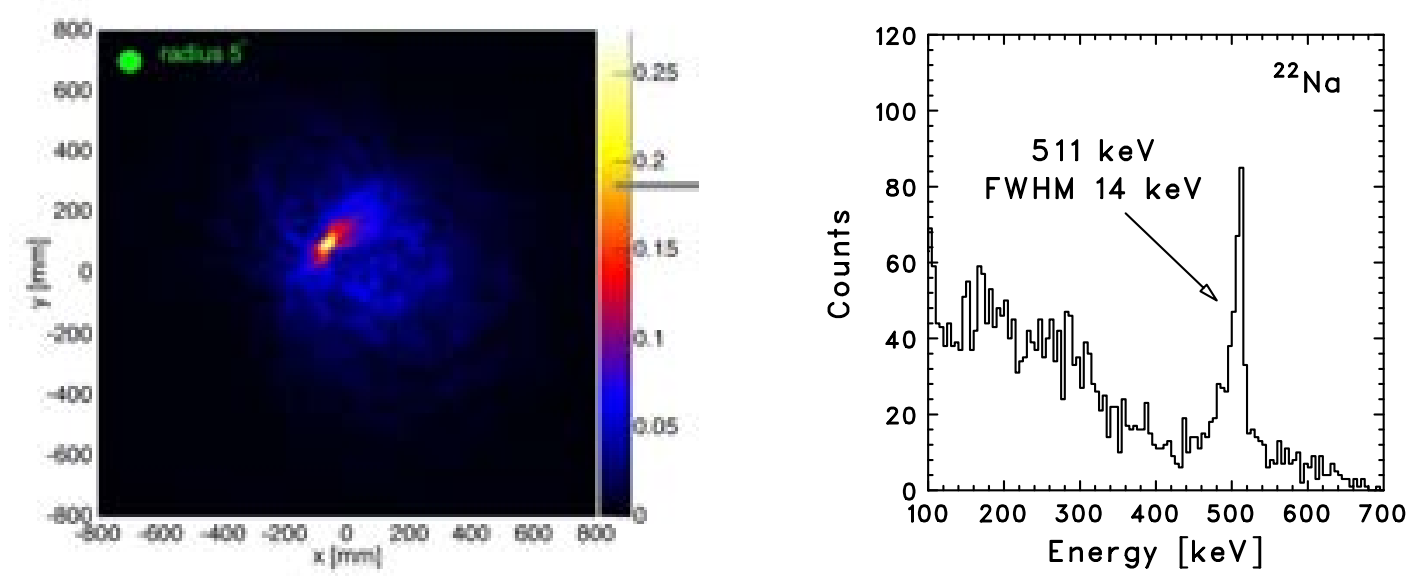

Figure 1. Compton reconstructed image (left) and spectrum (right) of the $511 \mathrm{keV}$ gamma-ray obtained with a prototype $\mathrm{Si} / \mathrm{CdTe}$ Compton telescope. The angular resolution is $3.9^{\circ}$. The energy resolution is $14 \mathrm{keV}$ (FWHM) at $511 \mathrm{keV}$.

the stacked detector, we developed a prototype, which is composed of three layers of CdTe pixel detectors along with one at their side. In this paper, the results obtained with the prototype are described. The comparison with the results obtained from a Compton telescope consists of DSSD and CdTe pixel detectors are also carried out.

\section{A SI/CDTE COMPTON TELESCOPE}

As described in our previous publications, ${ }^{8-10}$ we have constructed a prototype Si/CdTe Compton telescope with different configurations. The high energy resolution of a newly developed DSSD has proven to be essential to obtain not only a wide energy coverage starting from $60 \mathrm{keV}$, but also a high angular resolution. With the prototype $\mathrm{Si} / \mathrm{CdTe}$ Compton telescope, we obtained Compton reconstructed images of the gamma-rays from 81 $\mathrm{keV}$ to $662 \mathrm{keV}$. The $511 \mathrm{keV}$ image is shown in the Fig. 1. The angular resolution is measured to be $3.9{ }^{\circ}$ for $511 \mathrm{keV}$ gamma-rays. Fig. 1 shows the reconstructed spectrum for $511 \mathrm{keV}$ gamma-rays from a ${ }^{22} \mathrm{Na}$ gamma-ray source. The energy resolution of $14 \mathrm{keV}(\mathrm{FWHM})$ demonstrates the high potential of the Si/CdTe Compton telescope for future missions.

Since the thickness of the CdTe pixel detector adopted as an absorber in the prototype is only $0.5 \mathrm{~mm}$, the total efficiency for gamma-rays above several hundred $\mathrm{keV}$ is very limited. A possible improvement to this is to thicken the CdTe detector. A monolithic and thick detector is not, however, applicable if we use the current CdTe or CdZnTe semiconductor, due to incomplete charge collection. Therefore, we are working on the concept of a stacked CdTe detector with a capability of imaging.

\section{A STACKED THIN CDTE PIXEL DETECTOR FOR COMPTON TELESCOPE}

For gamma-rays above a hundred keV, adequate detection efficiency can be obtained by CdTe or CdZnTe with a thickness of more than $5 \mathrm{~mm}$. It is, however, difficult to increase the thickness of the device, keeping good energy resolution throughout the energy range from several ten $\mathrm{keV}$ to a few hundred $\mathrm{keV}$. This is because the effect of incomplete charge collection becomes more severe due to small mobility and short lifetime of holes of CdTe or CdZnTe. The inactive region in the detector volume degrades the energy resolution through a low energy tail in the spectral response. ${ }^{14,15}$

In order to achive fully active CdTe or CdZnTe detectors, the concept of using thin device is of particular importance, since we can apply sufficient bias voltage to collect all charge produced in the detector. As calculated in our paper, ${ }^{12}$ a $0.5 \mathrm{~mm}$ thick CdTe detector becomes fully active with a bias voltage of $800 \mathrm{~V}$, and even holes generated near the anode face can be completely collected. By using a detector with a thickness of $0.5 \mathrm{~mm}$, an 


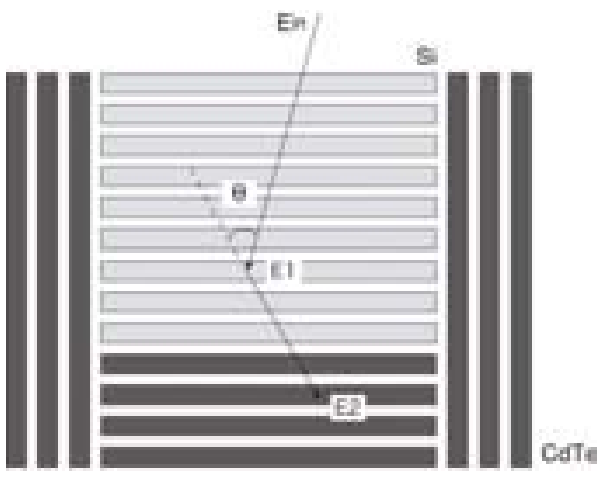

Figure 2. Conceptual drawing of our $\mathrm{Si} / \mathrm{CdTe}$ semiconductor Compton telescope. CdTe pixel detectors are stacked into 20 layers to achieve larger effective area.

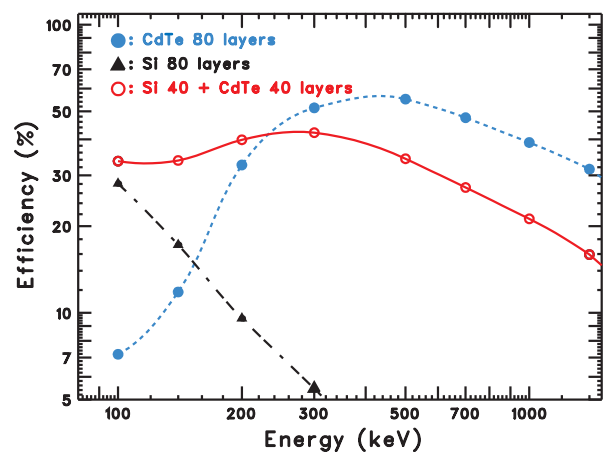

Figure 3. Calculated peak detection efficiency for three types of Compton telescopes. In the simulation, the detector is defined to consist of 80 layers of thin $\mathrm{Si}$ or $\mathrm{CdTe}$ detector. It can be clearly seen that the adoption of CdTe leads to high detection efficiency for a Compton telescope with stacked configuration. [Takahashi, et al, SPIE (2002)]

applied bias voltage of $1400 \mathrm{~V}$, and a temperature of $-20{ }^{\circ} \mathrm{C}$, we have achieved an energy resolution (FWHM) of $830 \mathrm{eV}$ at $59.5 \mathrm{keV}$, and $2.1 \mathrm{keV}$ at $662 \mathrm{keV}$, which is close to the Fano limit of CdTe. ${ }^{15}$

For energies up to a few $\mathrm{MeV}$, stacking thin CdTe pixel detectors becomes the unique concept to realize a detector with both high energy resolution and high efficiency for gamma-rays. ${ }^{12-14}$ As shown in Fig. 2, a stacked CdTe detector is adopted in our $\mathrm{Si} / \mathrm{CdTe}$ Compton telescope. In the stacked detector, several thin CdTe pixel detectors are stacked together and operated as a single detector. By adopting fully-active thin CdTe pixel detector in each layer, we can take advantage of the high energy resolution for gamma-rays from several tens of $\mathrm{keV}$ to a few $\mathrm{MeV}$.

Another important merit of utilizing a stacked thin CdTe pixel detector is that it works as an effective Compton telescope above $300 \mathrm{keV}$, because the stacked detector gives three-dimensional information of the gamma-ray interaction in the CdTe part. It should be noted that the cross section for Compton scattering in CdTe becomes higher than that of photo absorption above $300 \mathrm{keV}$, and the cross section is twice larger than that of Si. Fig. 3 clearly shows how the stacked CdTe works to improve the efficiency for the high energy gamma-rays. Larger efficiency for the gamma-rays above a hundred $\mathrm{keV}$ can be achieved by optimizing the number of layers of CdTe detectors. Although the angular resolution of a stacked CdTe pixel detector is expected to be worse than that of a $\mathrm{Si} / \mathrm{CdTe}$ Compton telescope due to the larger Doppler broadening effect, ${ }^{16}$ the high efficiency and high energy resolution are attractive for the detection of gamma-rays up to a few $\mathrm{MeV}$.

\section{EXPERIMENTAL SETUP}

In order to operate a stacked CdTe pixel detector as a Compton telescope, not only high resolution CdTe pixel detector but also an individual readout system is essential. In this section, details of the CdTe pixel detector and the detector system are described.

\subsection{High Resolution $8 \times 8$ CdTe Pixel Detectors}

The $8 \times 8$ CdTe pixel detector used in the prototype is based on the Schottky CdTe diode device, utilizing indium as the anode and platinum as the cathode. The CdTe crystal is manufactured by ACRORAD in Japan with the Traveling Heater Method (THM). It features very low leakage current and high uniformity. ${ }^{1,17}, 18$ Since position resolution of a few $\mathrm{mm}$ is necessary to construct a Compton telescope, we have developed a pixel detector with 64 pixels. Fig. 4 shows the photo of the detector. The detector has dimensions of $18.55 \mathrm{~mm} \times 18.55 \mathrm{~mm}$ and a thickness of $500 \mu \mathrm{m}$. The indium side is used as a common electrode and the platinum side is divided into 8 by 8 pixels. The pixel size is $2 \mathrm{~mm} \times 2 \mathrm{~mm}$, and the gap between each pixel is $50 \mu \mathrm{m}$. A guard ring electrode with a 


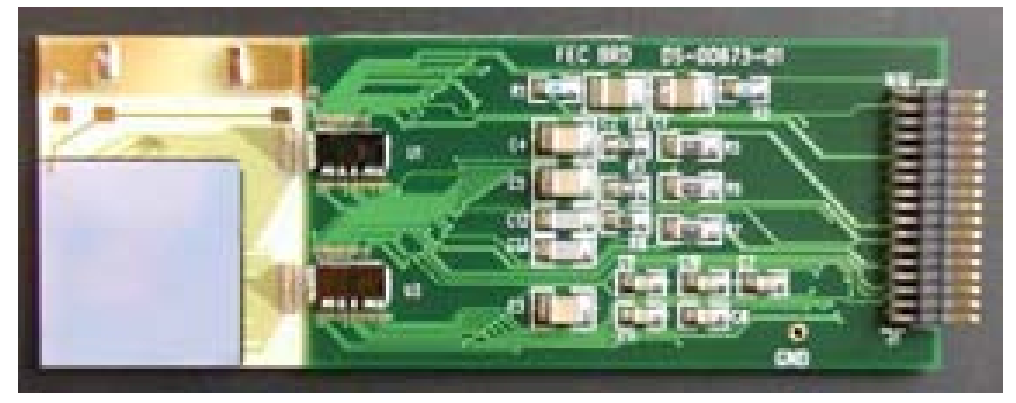

Figure 4. A photo of a $8 \times 8$ CdTe pixel detector. The signal from each pixel is fed into two VA32TAs on the FEC.

width of $1 \mathrm{~mm}$ is attached to reduce leakage current because most of the leakage current is through the detector perimeter. Each pixel is connected to a fanout board by bump bonding technology developed in cooperation with Mitsubishi Heavy Industry in Japan. ${ }^{14}$ Since the leakage current of each pixel is as low as $100 \mathrm{pA}$ at a bias voltage of $500 \mathrm{~V}$ even at room temperature, the signal lines are directly connected to the input of readout electronics, an analog LSI VA32TA. ${ }^{5}$ This LSI is developed with IDEAS in Norway, and characterized by its low noise. The LSIs are read out via a specially designed compact readout system including an ADC and FPGA, which is controlled with a fast serial interface "Space Wire (IEEE 1355). ${ }^{19}$ "

High energy resolution of each CdTe pixel detector is essential to achieve both high angular resolution and energy resolution for Compton telescopes. The low energy threshold of the detector is also important in order to utilize events with small-angle scattering. This is because the deposited energy of a recoiled electron becomes lower as the scattering angle becomes smaller. Fig. 5 shows spectra of ${ }^{22} \mathrm{Na}$ and ${ }^{57} \mathrm{Co}$ with one of the $\mathrm{CdTe}$ pixel detectors employed in the prototype stacked CdTe pixel detector. The spectra are drawn by summing the spectra from all channels after gain correction. The data is measured at a temperature of $-20{ }^{\circ} \mathrm{C}$ and with a bias voltage of $1200 \mathrm{~V}$. The energy resolution (FWHM) is $5.0 \mathrm{keV}$ and $1.6 \mathrm{keV}$ for the gamma-rays of $511 \mathrm{keV}$ and $122 \mathrm{keV}$, respectively. The energy threshold is measured to be as low as $7 \mathrm{keV}$.

The homogeneity of the wafer is of particular importance for imaging detectors, because defects in the wafer can deteriorate the energy resolution through positional variation of properties such as charge collection efficiency and leakage current. As reported in our previous publications, ${ }^{1,18}$ we have demonstrated the uniform response of large THM-CdTe detectors with an area of $21 \mathrm{~mm} \times 21 \mathrm{~mm}$. Fig. 6 shows spectra of ${ }^{22} \mathrm{Na}$ for each channel. The same data as in Fig. 5 (left) is used. In this detector, all 64 channels are examined to be properly connected, and the detector shows uniform response.

\subsection{Prototype Setup of CdTe Compton Telescope}

Fig. 7 shows the arrangement of the detectors. Three CdTe pixel detectors are stacked with an interval of 12 $\mathrm{mm}$, while one detector are placed at the side of them. In order to lower the leakage current and to surpress the so-called "polarization effect", ${ }^{20}$ the entire detector system was kept at a temperature of $-20{ }^{\circ} \mathrm{C}$, and the applied bias voltage for the all detectors was set to $700 \mathrm{~V}$. Under these conditions, we do not see any spectral degradation for a long term operation of a week, as is reported in our paper. ${ }^{1}$ Gamma-ray sources are placed $370 \mathrm{~mm}$ above the surface of the first layer of the detector. A trigger signal is issued from all channels unless it is disabled due to large noise. When a trigger signal is generated from some channels, all channels of the entire system are read out.

\section{DATA ANALYSIS}

For Compton reconstruction, data reduction is performed as follows. First, "two-hit events," one hit in a CdTe pixel detector and one hit in the other detector, were selected from the raw data. Here, one hit means that only one channel has a pulse height above $14 \mathrm{keV}$. Fig. 8 shows an example of a scatter plot for the "two-hit events." This plot is obtained when a ${ }^{22} \mathrm{Na}$ radiative isotope is used. There is a branch which satisfies $\mathrm{E} \sim 511 \mathrm{keV}$, 

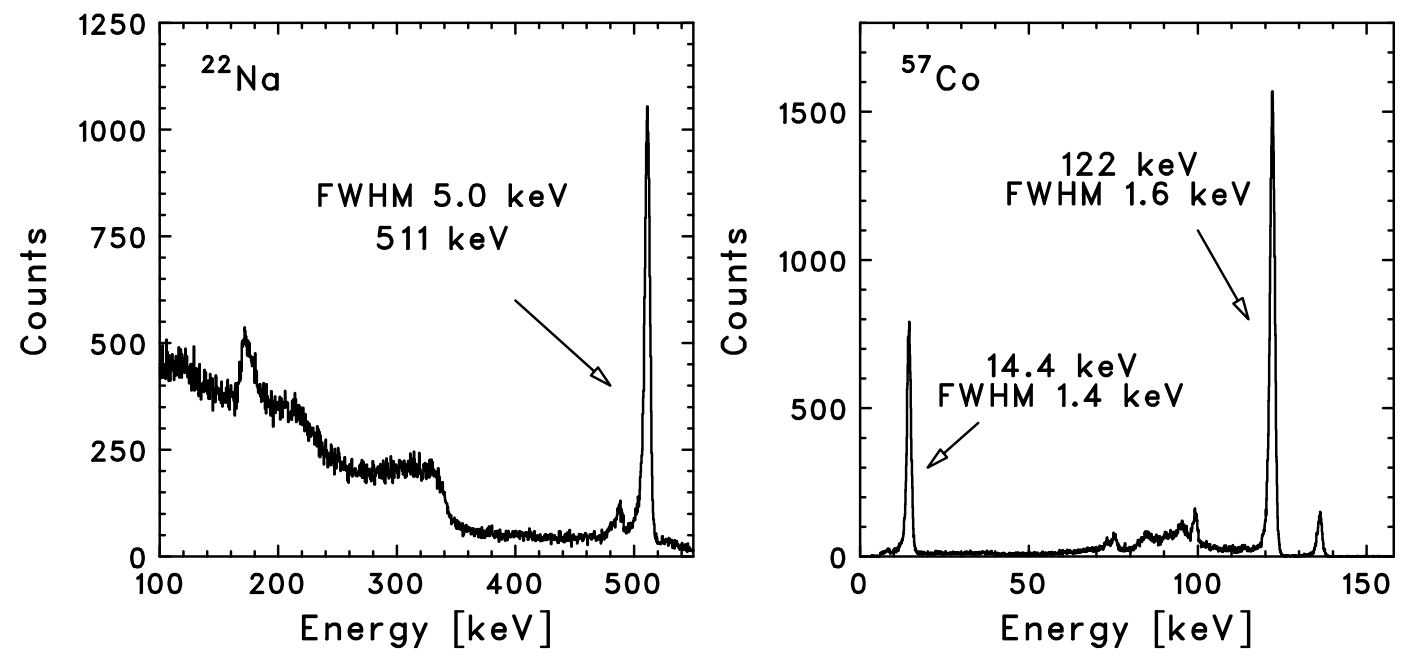

Figure 5. ${ }^{22} \mathrm{Na}$ (left) and ${ }^{57} \mathrm{Co}$ spectra obtained with a $8 \times 8 \mathrm{CdTe}$ pixel detector. The detector is operated at a temperature of $-20{ }^{\circ} \mathrm{C}$, with a bias voltage of $1200 \mathrm{~V}$. Each spectrum is drawn by summing spectra of all 64 channels. The energy resolution (FWHM) at $511 \mathrm{keV}$ and $122 \mathrm{keV}$ is $5.0 \mathrm{keV}$ and $1.6 \mathrm{keV}$, respectively.

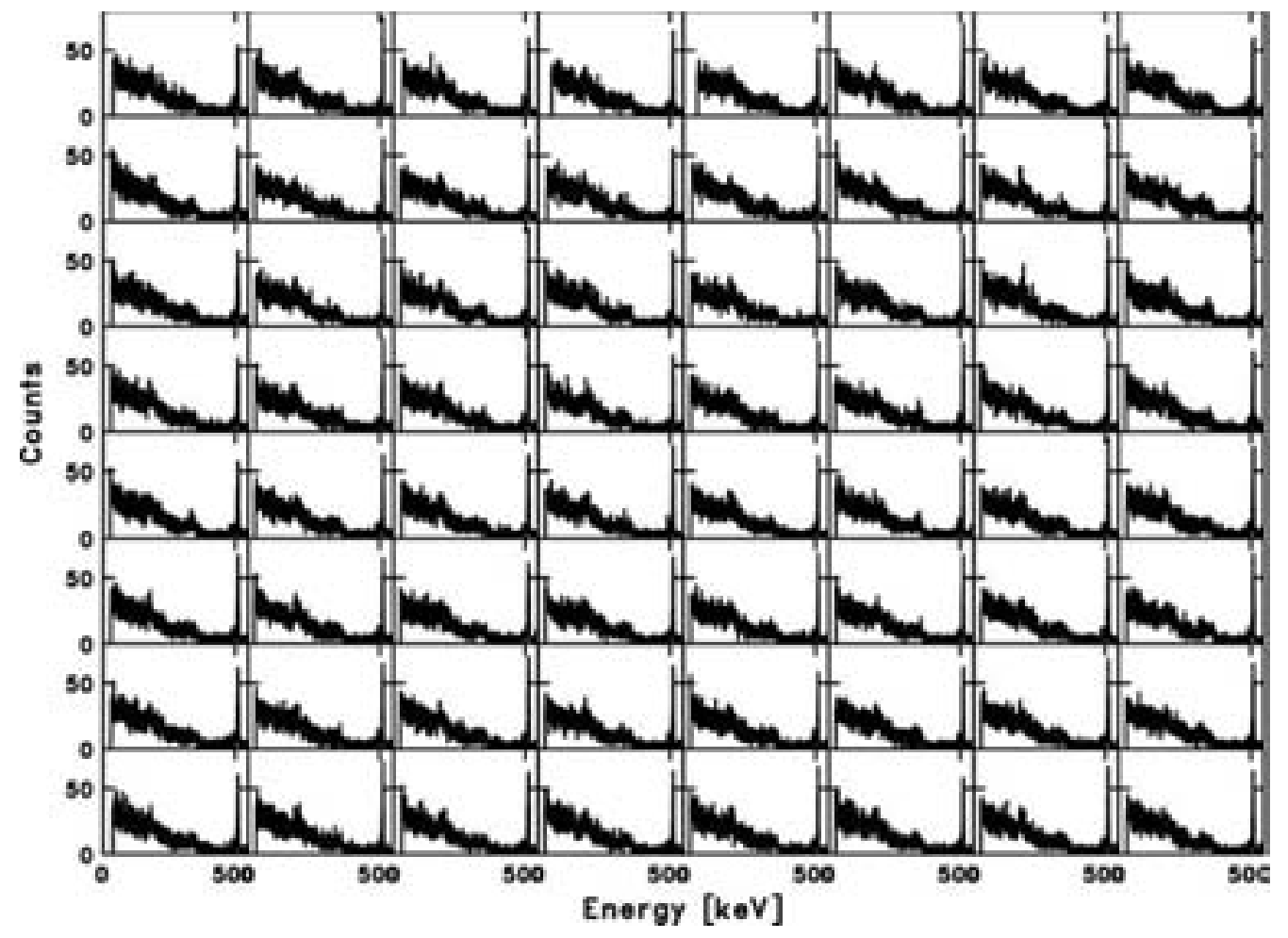

Figure 6. $511 \mathrm{keV}$ spectra of all the 64 pixels. 


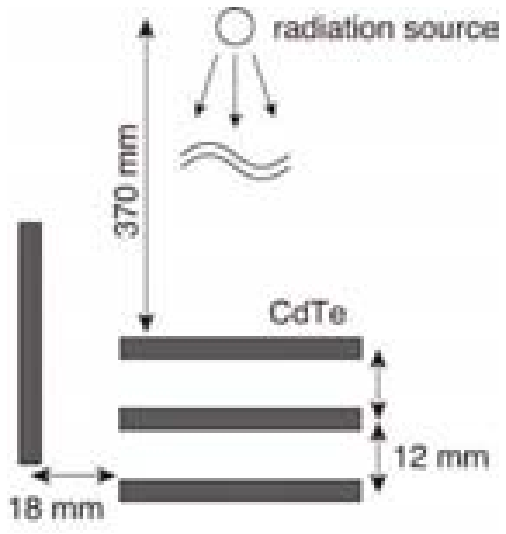

Figure 7. Configuration of the prototype stacked CdTe pixel detector.

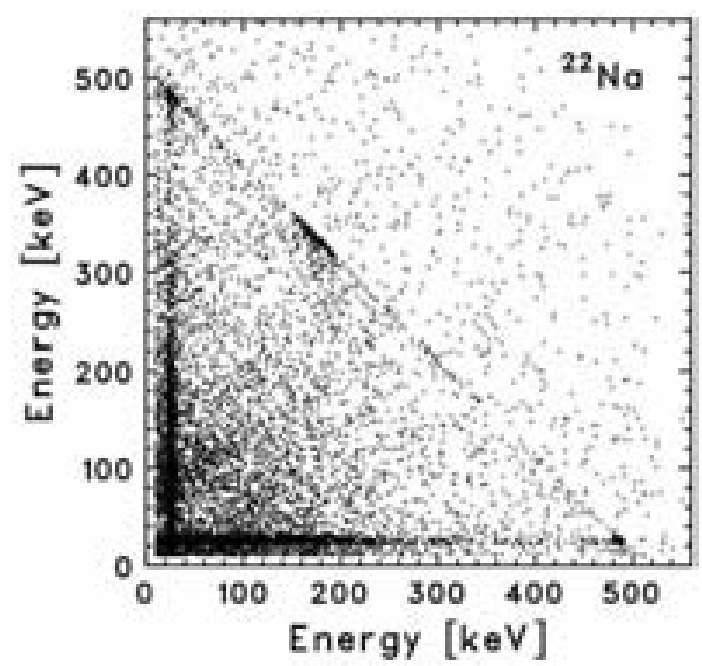

Figure 8. Scatter plot of all two-hit events. The plot is obtained by using a ${ }^{22} \mathrm{Na}$ gamma-ray source.

which means incident gamma-rays of $511 \mathrm{keV}$ are scattered in one detector and absorbed in the other. Other noticeable branches are those parallel to the horizontal axis and to the vertical axis between $20 \mathrm{keV}$ and $34 \mathrm{keV}$, respectively. They are the events due to fluorescence X-rays from $\mathrm{Cd}(\mathrm{K} \alpha: 23.1 \mathrm{keV}, \mathrm{K} \beta: 26.1 \mathrm{keV})$ and $\mathrm{Te}(\mathrm{K} \alpha$ : $27.4 \mathrm{keV}, \mathrm{K} \beta: 31.0 \mathrm{keV}$ ), which escape from the CdTe pixel detectors and are absorbed in the other. Since they are not the Compton events, we eliminate those events in the analysis.

From each two-hit event, the information of deposited energies and positions are obtained. We name the smaller deposited energy as $E_{1}$, and the other as $E_{2}\left(E_{1} \leq E_{2}\right)$. Using these energies, the incident energy $\left(E_{\text {in }}\right)$ and the scattering angle $\left(\theta_{\text {comp }}\right)$ are calculated. In the calculation, we used:

$$
\begin{aligned}
E_{i n} & =E_{1}+E_{2} \\
\cos \theta_{\text {comp }} & =1-\frac{m_{e} c^{2}}{E_{2}}+\frac{m_{e} c^{2}}{E_{1}+E_{2}},
\end{aligned}
$$

assuming that incident gamma-rays are once scattered and then fully absorbed in the CdTe pixel detector. Eq. 2 assumes that a incident gamma-ray first deposits its energy $\left(E_{1}\right)$ by a Compton scattering, and then deposits the rest of the energy $\left(E_{2}\right)$ by photo absorption. If the energy of incident gamma-rays $\left(E_{i n}\right)$ is less than $\mathrm{m}_{e} \mathrm{c}^{2} / 2$ $=255.5 \mathrm{keV}$, this assumption is valid because the energy of the recoil electron is always less than that of the scattered photon. If the energy of the incident gamma-rays exceeded $255.5 \mathrm{keV}$, we calculated two scattering angles by swapping $E_{1}$ and $E_{2}$ in Eq. 2. From the calculated scattering angle and the hit positions, Compton cones are drawn on the sky event by event. The Compton cones are weighted by using the differential cross section of Compton scattering using the Klein-Nishina formula, and the cross section of photo absorption. We projected the cone at the plane at the distance of $370 \mathrm{~mm}$, and obtained the image of the gamma-ray sources.

\subsection{Compton Reconstruction of Images}

Fig. 9 shows the images obtained with four gamma-ray sources. A circle with a radius of $10^{\circ}$ is drawn together with each image as a reference. Fig. 9 (a) is the image of ${ }^{57} \mathrm{Co}$ gamma-rays, by using events within $122 \mathrm{keV} \pm$ $5 \mathrm{keV}$. The images of ${ }^{133} \mathrm{Ba},{ }^{22} \mathrm{Na}$, and ${ }^{137} \mathrm{Cs}$ are also drawn in Fig. 9 (b) - (d). The energy selection is $270 \mathrm{keV}$ $-390 \mathrm{keV}$ for ${ }^{133} \mathrm{Ba}, 500 \mathrm{keV}-520 \mathrm{keV}$ for ${ }^{22} \mathrm{Na}$, and $650 \mathrm{keV}-675 \mathrm{keV}$ for ${ }^{137} \mathrm{Cs}$, respectively. We note that the images are symmetric due to the arrangement of the CdTe pixel detectors being symmetric except for the one at a side. 


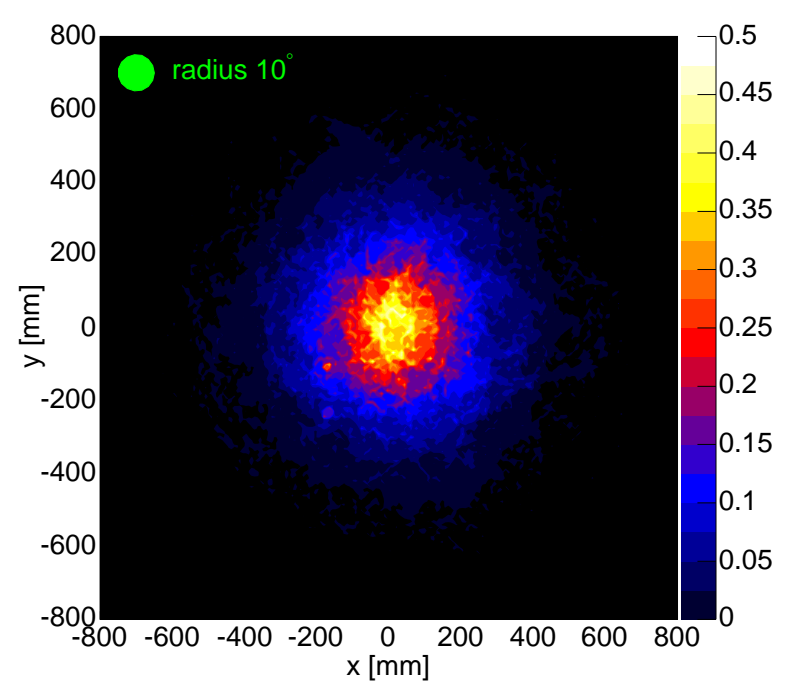

(a) ${ }^{57} \mathrm{Co}: 122 \mathrm{keV}$

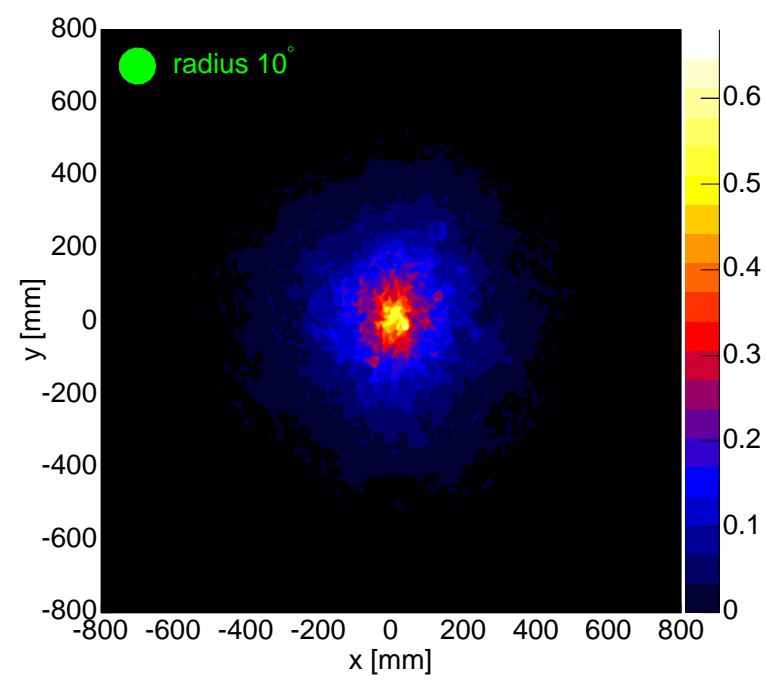

(c) ${ }^{22} \mathrm{Na}: 511 \mathrm{keV}$

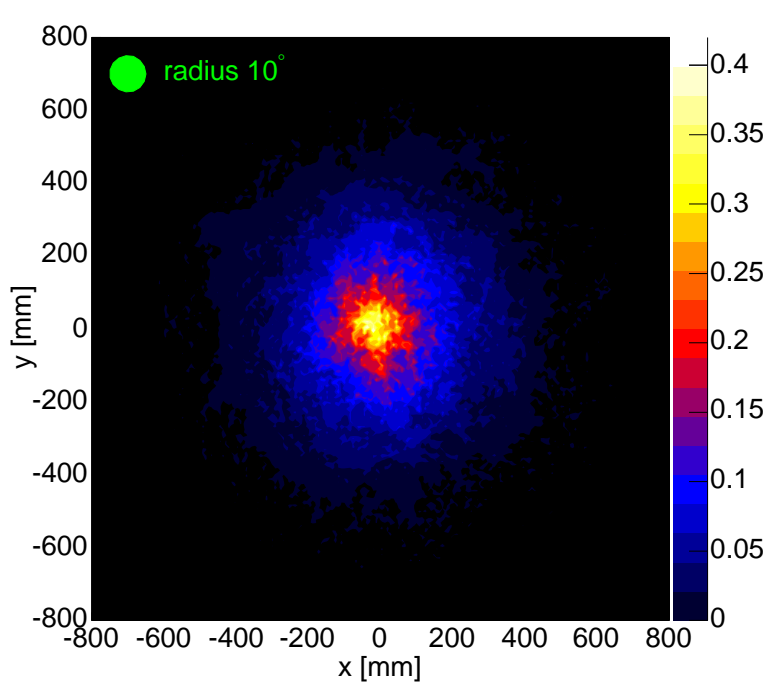

(b) ${ }^{133} \mathrm{Ba}: 276,303,356,384 \mathrm{keV}$

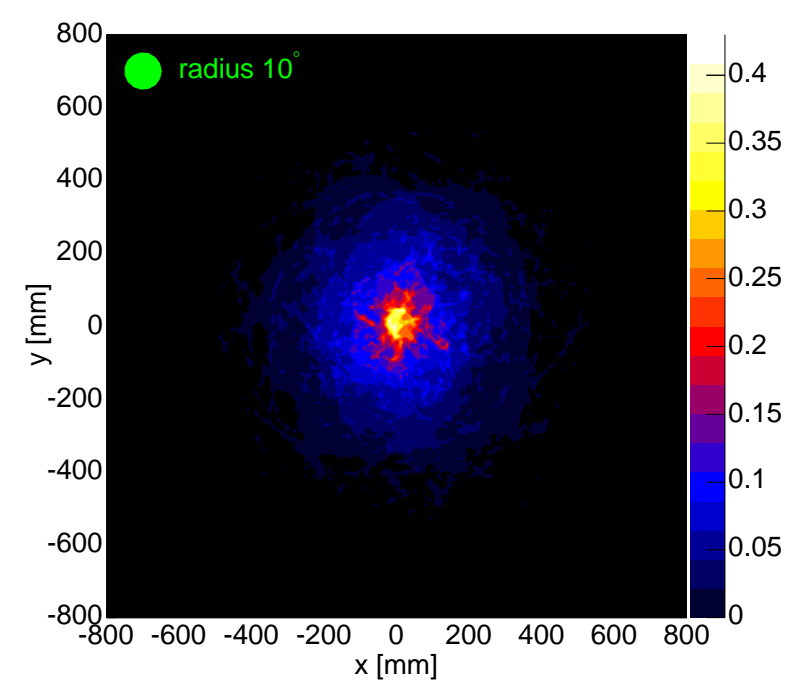

(d) ${ }^{137}$ Cs: $662 \mathrm{keV}$

Figure 9. Compton reconstructed images. (a) ${ }^{57} \mathrm{Co}$ image using $122 \mathrm{keV} \pm 5 \mathrm{keV}$. (b) ${ }^{133} \mathrm{Ba}$ image using $270 \mathrm{keV}-390$ $\mathrm{keV}$. (c) ${ }^{22} \mathrm{Na}$ image using $500 \mathrm{keV}-520 \mathrm{keV}$. (d) ${ }^{137} \mathrm{Cs}$ image using $650 \mathrm{keV}-675 \mathrm{keV}$. 

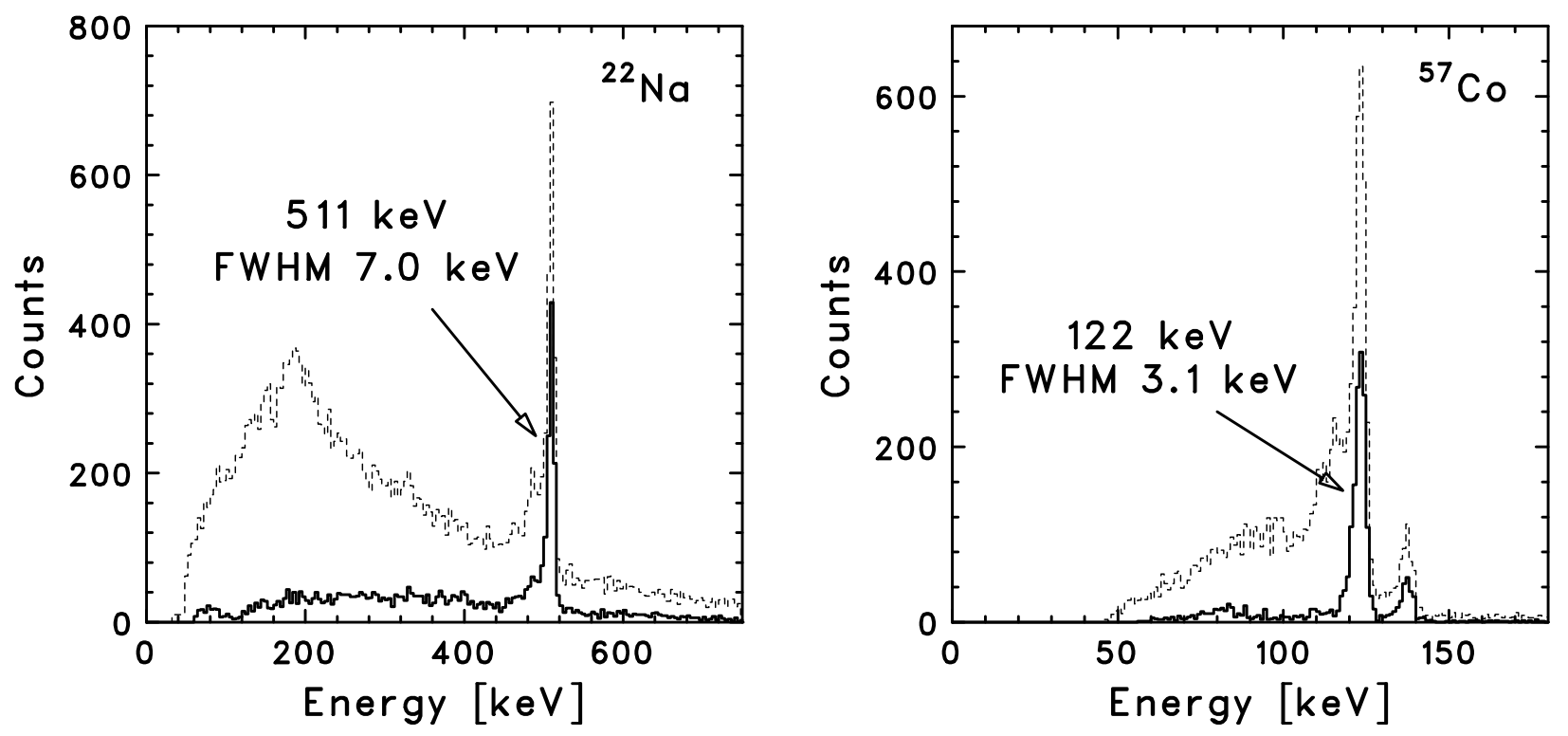

Figure 10. Compton reconstructed spectra of ${ }^{22} \mathrm{Na}$ (left) and ${ }^{57} \mathrm{Co}$ (right). Dotted lines show the simple sum of two-hit events, while solid lines show the sum of events identified to be emitted from the source by Compton reconstruction.

\subsection{Compton Reconstruction of Spectra}

Background rejection using a Compton image is an important feature for a Compton telescope. Energy spectra of the incident gamma-rays are obtained by summing deposited energies. The dotted lines in Fig. 10 shows the spectra which we obtained by simply summing the deposited energies at each detector for all "two-hit events." In the spectrum obtained with a ${ }^{22} \mathrm{Na}$ source, there is a $511 \mathrm{keV}$ gamma-ray line and a large number of scattering components. The latter components are considered as the events scattered twice in the CdTe pixel detectors, the events by the gamma-rays scattered in the other materials, and so forth. Then we performed an event selection using the Compton images. The solid line spectrum in Fig. 10 is made from the events which satisfy the condition that the drawn Compton cone corresponds to the direction of the source position. As shown in the figure, the peak of the $511 \mathrm{keV}$ gamma-rays are enhanced, and the scattering components are reduced. The energy resolution (FWHM) is $7.3 \mathrm{keV}$ for $511 \mathrm{keV}$ gamma-rays and $3.1 \mathrm{keV}$ for $122 \mathrm{keV}$ gamma-rays, respectively.

\subsection{Angular Resolution of the CdTe compton Telescope}

In order to evaluate the angular resolution of our prototype Compton telescope, we compared the calculated scattering angles $\left(\theta_{\text {comp }}\right)$ with those defined by the location of gamma-ray source and hit positions $\left(\theta_{\text {geom }}\right)$ (see Fig. 11). The difference between the two values reflects the angular resolution $\left(\Delta \theta=\theta_{\text {comp }}-\theta_{\text {geom }}\right)$ of the Compton telescope. Fig. 12 shows an example of events within $270 \mathrm{keV}-390 \mathrm{keV}$ for the data obtained with ${ }^{133} \mathrm{Ba}$. We also investigate the distribution of the angular resolution for the gamma-rays of ${ }^{57} \mathrm{Co},{ }^{22} \mathrm{Na}$, and ${ }^{137} \mathrm{Cs}$. The FWHM of these distributions $\left(\Delta \theta_{\text {exp }}\right)$, which is the angular resolution of the prototype, are plotted against the incident gamma-ray energies in Fig. 13. The angular resolution becomes better as the incident gamma-ray energy become higher. The obtained angular resolution is $35.9^{\circ}$ at $122 \mathrm{keV}$, and $12.2^{\circ}$ at $511 \mathrm{keV}$, respectively.

We estimated the contribution of the position and energy resolution of the CdTe pixel detectors. The contribution of the position resolution $\left(\Delta \theta_{p o s}\right)$ is estimated by using the actual hit positions obtained from the experiment, and the pixel size of the detectors. To estimate the contribution of the energy resolution $\left(\Delta \theta_{\text {ene }}\right)$, we used the Compton-scattering angles of actual events and smoothed them by the energy resolution of the detector. The estimated values are plotted in Fig. 13. The other contribution to the angular resolution is the effect of the Doppler broadening. In order to estimate the amount of them $\left(\Delta \theta_{D B}\right)$, we performed Monte Carlo simulations 


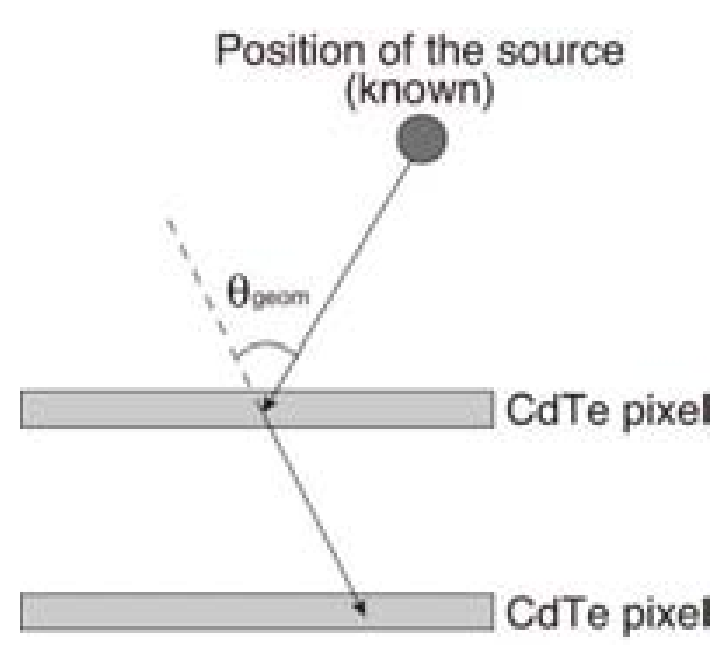

Figure 11. Definition of $\theta_{\text {geom }}$.

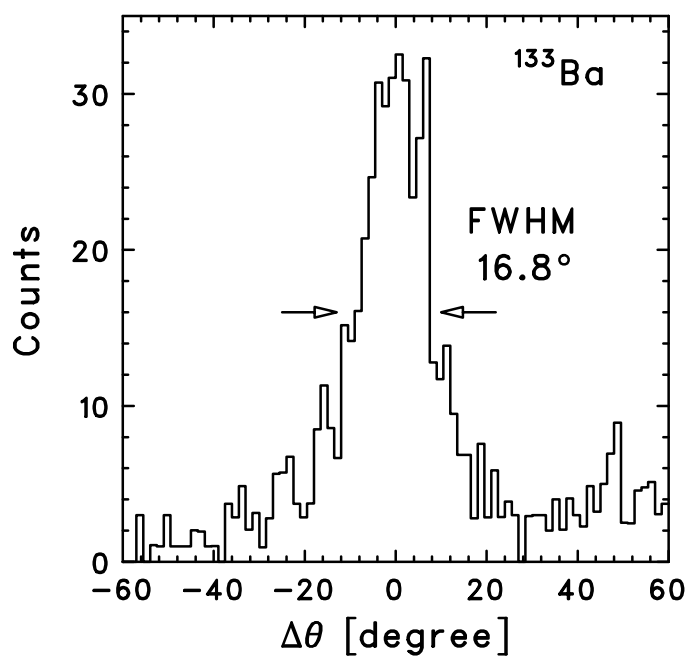

Figure 12. Distribution of $\theta_{\text {comp }}-\theta_{\text {geom }}$ for ${ }^{133}$ Ba gammaray source.

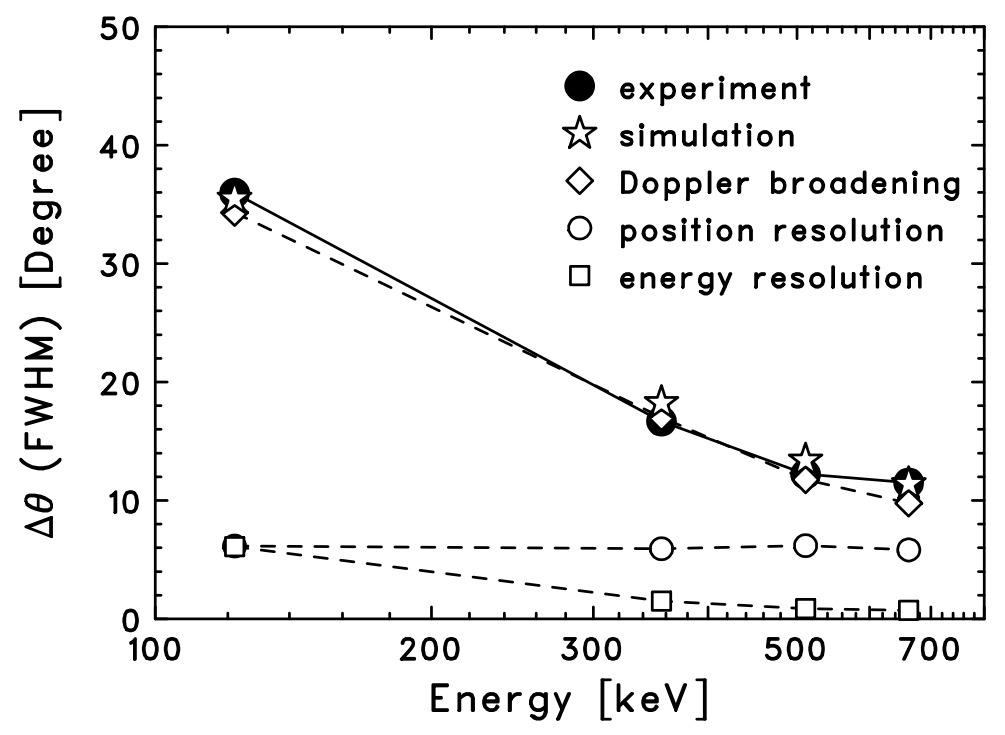

Figure 13. Relation between incident gamma-ray energy and angular resolution (FWHM). Filled circles show the experimental data. Diamonds, open circles, and squares are estimated contributions of Doppler broadening, position resolution, and energy resolution, respectively. The total values of the three are ploted by stars.

using the Geant4 package, ${ }^{21}$ and plotted them in the same figure. The effect of the Doppler broadening becomes smaller as the incident energy becomes higher, which is consistent with the paper. ${ }^{16}$ The total value $\left(\Delta \theta_{\text {total }}\right)$ is also plotted in Fig. 13, which is calculated from:

$$
\Delta \theta_{\text {total }}=\sqrt{\left(\Delta \theta_{\text {pos }}\right)^{2}+\left(\Delta \theta_{\text {ene }}\right)^{2}+\left(\Delta \theta_{D B}\right)^{2}} .
$$

The experimental values can be explained with the three contributions. The angular resolution is almost limited by the effect of Doppler broadening. 


\section{CONCLUSIONS}

A Si/CdTe semiconductor Compton telescope is a promising detector for future gamma-ray missions in the energy band from several tens of $\mathrm{keV}$ to a few $\mathrm{MeV}$. In order to achieve higher efficiency, we have verified a concept of a stacked CdTe pixel detector which acts as both a scatterer and an absorber. The prototype detector consists of three layers of CdTe pixel detectors and one CdTe pixel detector at their side. With this prototype detector, we succeeded in Compton reconstruction of images and spectra in the energy band from $122 \mathrm{keV}$ to $662 \mathrm{keV}$. The energy resolution (FWHM) of reconstructed spectra is $7.3 \mathrm{keV}$ at $511 \mathrm{keV}$ and $3.1 \mathrm{keV}$ at 122 $\mathrm{keV}$, respectively. The high energy resolution of the reconstructed spectra is due to the high performance of thin CdTe pixel detectors. The obtained angular resolution is $35.9^{\circ}$ at $122 \mathrm{keV}$, and $12.2^{\circ}$ at $511 \mathrm{keV}$, which is mainly dominated by the effect of Doppler broadening.

The prototype stacked CdTe pixel detector shows higher efficiency in terms of reconstructed spectra than that of the prototype $\mathrm{Si} / \mathrm{CdTe}$ Compton telescope, while angular resolution of the stacked CdTe is worse than that of the prototype $\mathrm{Si} / \mathrm{CdTe}$ Compton telescope below $1 \mathrm{MeV}$. However, the angular resolution becomes a degree scale, when the gamma-ray energy goes up to a few MeV. Once the stacked CdTe pixel detector is combined with Si layers, the detector should give us a good performance from several $10 \mathrm{keV}$ up to several MeV.

\section{REFERENCES}

1. T. Takahashi, T. Mitani, Y. Kobayashi, M. Kouda, G. Sato, S. Watanabe, K. Nakazawa, Y. Okada, M. Funaki, R. Ohno, and K. Mori, "High Resolution Schottky CdTe Diodes," IEEE Trans. Nucl. Sci., 49, No. 3, pp. 1297-1303, 2002

2. T. Tanaka, et al., "Recent achievements of the high resolution Schottky CdTe diode for $\gamma$-ray detectors," New Astronomy Reviews, vol. 48, pp. 309-313, 2004

3. K. Nakazawa, K. Oonuki, T. Tanaka, Y. Kobayashi, K. Tamura, T. Mitani, G. Sato, S. Watanabe, T . Takahashi, R. Ohno, A. Kitajima, Y. Kuroda and M. Onishi "Improvement of the CdTe Diode Detectors using a Guard-ring Electrode," IEEE Trans. Nucl. Sci, in press, 2004

4. Y. Fukazawa, et al., "Low noise Double-sided Silicon Strip Detector for Soft Gamma-ray Compton Camera," Proc SPIE, vol. 5501, 2004

5. H. Tajima, et al, "Low Noise Double-Sided Silicon Strip Detector for Multiple-Compton Gamma-ray Telescope," Proc. SPIE, vol. 4851, pp. 875-884, 2003

6. T. Takahashi, K. Makishima, F. Fukazawa, M. Kokubun, K. Nakazawa, M. Nomachi, H. Tajima, M. Tashiro, and Y. Terada, "Hard X-ray and Gamma-ray detectors for the next mission,", New Astronomy Reviews, vol 48, pp. 309-313, 2004

7. T. Takahashi, K. Nakazawa, T. Kamae, H. Tajima, Y. Fukazawa, M. Nomachi, and M. Kokubun , "High resolution CdTe detectors for the next generation multi-Compton gamma-ray telescope " Proc. SPIE, vol. 4851, pp. 1228-1235, 2002

8. T. Mitani, T. Tanaka, K. Nakazawa, T. Takahashi, T. Takashima, H. Tajima, H. Nakamura, M. Nomachi, T. Nakamoto, Y. Fukazawa "A Prototype Si/CdTe Compton Camera and the Polarization Measurement", IEEE Trans. Nucl. Sci., in press, 2004

9. T. Tanaka et al., "Development of a Si/CdTe semiconductor Compton Telescope," Proc. SPIE, vol. 5501, 2004

10. S. Watanabe, et al., "A Si/CdTe Semiconductor Compton Camera," IEEE Trans. Nucl. Sci., in press, 2005

11. T. Takahashi, B. Paul, K. Hirose, C. Matsumoto, R. Ohno, T. Ozaki, K. Mori, and Y. Tomita, "Highresolution Schottky CdTe Detectors for Hard X-ray and Gamma-ray Observations," Nucl. Instrum. Methods, vol. A436, pp. 111-119, 2000

12. S. Watanabe, et al., "CdTe stacked detectors for Gamma-ray detection," IEEE Trans. Nucl. Sci., vol. 49, pp. 1292-1296, 2001

13. S. Watanabe, et al., "Stacked CdTe Gamma-ray Detector and its application to a range finder," Nucl. Instr. Methodes, vol. A 505, pp. 118-121, 2003

14. T. Takahashi, et al., "High-Resolution CdTe Detector and Applications to Imaging Devices", IEEE Trans. Nucl. Sci., 48, pp. 287-291, 2001 
15. T. Takahashi and S. Watanabe "Recent Progress on CdTe and CdZnTe detectors," IEEE Trans. Nucl. Sci., vol. 48, pp. 950-959, 2001

16. A. Zoglauer and G. Kanbach, "Doppler Broadening as a Lower Limit to the Angular Resolution of Next Generation Compton Telescopes," Proc SPIE, vol. 4851, pp. 1302-1309, 2003

17. M. Funaki, et al., "Growth and Characterization of CdTe Single Crystal for Radiation Detectors," Nucl. Instrum. Methods., vol. A436, pp. 120-126, 1999

18. K. Nakazawa, T. Takahashi, S. Watanabe, G. Sato, M. Kouda, Y. Okada, T. Mitani, Y. Kobayashi, Y. Kuroda, M. onishi, R. Ohno, and A. Kitajima, "Large-area CdTe diode detector for space application," Nucl. Instrum. Methods, vol. A512, pp. 412-418, 2003

19. T. Mitani, et al., "Large area Gamma-ray Imaging Detector Based on High Resolution CdTe Diode," IEEE Trans. Nucl. Sci., vol. 50, pp. 412-418, 2003

20. T. Takahashi, K. Hirose, C. Matsumoto, K. Takizawa, R. Ohno, T. Ozaki, K. Mori, and Y. Tomita, "Performance of a New Schottky CdTe Detector for Hard X-ray Spectroscopy," Proc. SPIE, vol. 3446, pp. 29-37, 1998

21. R. M. Kippen, "The GEANT Low Energy Compton Scattering (GLECS) Packages for use in Simulating Advanced Compton Telescopes," New Astronomy Reviews, vol. 48, pp. 221-225, 2004 